\title{
Evaluation of uptake and transport of cationic and anionic ultrasmall iron oxide nanoparticles by human colon cells
}

This article was published in the following Dove Press journal:

International Journal of Nanomedicine

3 March 2012

Number of times this article has been viewed

\author{
Blanka Halamoda Kenzaoui' \\ Maya R Vilà ${ }^{2}$ \\ Josep M Miquel ${ }^{2}$ \\ Feride Cengelli' \\ Lucienne Juillerat- \\ Jeanneret ${ }^{\prime}$ \\ 'Centre Hospitalier Universitaire \\ Vaudois (CHUV) and University \\ of Lausanne (UNIL), Lausanne, \\ Switzerland; ${ }^{2}$ Leitat Technological \\ Center, Barcelona, Spain
}

Correspondence: Lucienne JuilleratJeanneret

University Institute of Pathology,

rue du Bugnon 25, $\mathrm{CH}$ - I0I I Lausanne,

Switzerland

Tel $+4|2| 3|47| 73$

Fax $+4|2| 3|47| 15$

Email lucienne.juillerat@chuv.ch

\begin{abstract}
Nanoparticles (NPs) are in clinical use or under development for therapeutic imaging and drug delivery. However, relatively little information exists concerning the uptake and transport of NPs across human colon cell layers, or their potential to invade three-dimensional models of human colon cells that better mimic the tissue structures of normal and tumoral colon. In order to gain such information, the interactions of biocompatible ultrasmall superparamagnetic iron oxide nanoparticles (USPIO NPs) (iron oxide core 9-10 nm) coated with either cationic polyvinylamine (aminoPVA) or anionic oleic acid with human HT-29 and Caco-2 colon cells was determined. The uptake of the cationic USPIO NPs was much higher than the uptake of the anionic USPIO NPs. The intracellular localization of aminoPVA USPIO NPs was confirmed in HT-29 cells by transmission electron microscopy that detected the iron oxide core. AminoPVA USPIO NPs invaded three-dimensional spheroids of both HT-29 and Caco-2 cells, whereas oleic acid-coated USPIO NPs could only invade Caco-2 spheroids. Neither cationic aminoPVA USPIO NPs nor anionic oleic acid-coated USPIO NPs were transported at detectable levels across the tight CacoReady ${ }^{\mathrm{TM}}$ intestinal barrier model or the more permeable mucus-secreting CacoGoblet ${ }^{\mathrm{TM}}$ model.
\end{abstract}

Keywords: iron oxide nanoparticles, human colon cells, spheroids, transport, gastrointestinal barrier

\section{Introduction}

Recent developments of diagnostic and therapeutic technologies in medicine include nanotechnologies to improve the early detection and treatment of human diseases. ${ }^{1,2}$ However, there is an urgent need to better understand the mechanisms of the interaction of nanomaterials, including nanoparticles (NPs), with living tissues, and to define the consequences of these interactions in order to assess the potential risks associated with nanotechnologies. In particular, there is a need to define the potential of NPs to be internalized by representative cells of tissues, or to be transported across biological cell barriers or inside three-dimensional models of either normal or diseased tissues for drug delivery and/or theranostic procedures. The development and use of nanomaterials by industry and for therapeutic purposes elevates the potential of human exposure through various routes, including the gastrointestinal tract.

Ultrasmall super paramagnetic iron oxide nanoparticles (USPIO NPs) were developed for magnetic resonance imaging (MRI) of the reticuloendothelial system such as the liver, spleen and lymph nodes, for perfusion imaging of the brain, myocardium, and kidney, and for angiography and tumor vascular imaging. ${ }^{3-5}$ Dextran-coated USPIO NPs are now in clinical use for disease detection by MRI. 
However, there are few reported precedents of the evaluation of the uptake and transport of USPIO NPs by human colon cells in two-dimensional barrier models or three-dimensional cellular models, the intracellular localization of these USPIO NPs following uptake, or their effect on the intestinal barrier following exposure. ${ }^{6}$

Multiple applications of USPIO NPs with different coatings are under development as new contrast and theranostic agents for colon and especially colon cancer imaging and treatment. ${ }^{7-9}$ The imaging purpose that these NPs will be used for determines whether they will be required to be able to cross biological barriers. To transport therapeutic agents and achieve intracellular drug delivery, USPIO NPs must be able to be internalized and transported by colon cells into deep layers of the tissue. Surface properties, charge, size, shape, and the engineered surface functionalization of NPs are important for their interactions (such as cell uptake, transport, or stress reactions) with cells. ${ }^{10,11}$ In particular, surface charges and biochemical properties of NPs will determine the adsorption of biological molecules, the "corona."12

The present study focused on the interactions between USPIO NPs possessing cationic or anionic surface coatings and human colon carcinoma cells. The USPIO NPs' potential to be taken up by monolayers of the colon cells was investigated, as was their ability to invade three-dimensional cell spheroid structures of the colon cells. Using electron microscopy to detect the NPs' iron oxide cores, it was possible to determine their cellular localization. The effect of USPIO NPs on colon barrier integrity was demonstrated using two different models of the gastrointestinal barrier, and the probability of their transport across these intestinal barrier models was evaluated.

\section{Methods USPIO NPs}

Oleic acid-coated ultrasmall superparamagnetic iron oxide USPIO NPs (oleic acid-coated USPIO NPs) are commercially available and were provided with physicochemical characterization by the provider. Oleic acid-coated USPIO NPs $\left(\mathrm{Fe}_{3} \mathrm{O}_{4}, 3 \%\right.$ oleic acid coating) were obtained from PlasmaChem (PlasmaChem GmbH, Berlin, Germany) as an approximately $7 \%$ nanosuspension in water, with average particle iron oxide core size $8 \pm 3 \mathrm{~nm}$, hydrodynamic size 14-15 nm (determined by DLS), and zeta potential $-30 \mathrm{mV}$ at pH 7 (values provided by PlasmaChem). The size of the iron oxide core was confirmed by transmission electron microscopy (data not shown). The size of oleic acid-coated
NPs in DMEM plus FCS cell culture medium displayed a bimodal size distribution of 25 and $150 \mathrm{~nm}$ as determined by DLS, and this size was stable toward further agglomeration for 3 days (information provided by D Bilanicova and $G$ Pojana, University of Venice, Italy; M Dusinska and the NanoTEST Consortium, manuscript in preparation).

The preparation and physicochemical characterization of polyvinylalcohol/polyvinylamine USPIO NPs (aminoPVA USPIO NPs) has previously been described. ${ }^{13-15}$ Briefly, ferrofluid was prepared by alkaline co-precipitation of ferric and ferrous chlorides, reflux in nitric oxide-ferrous nitrate, and dialysis providing iron oxide nanoparticles (ferrofluid) of $9 \mathrm{~nm}$. To obtain aminoPVA-coated USPIO NPs the ferrofluid was mixed with poly(vinylalcohol) (PVA), poly(vinylalcohol/ vinylamine)(aminoPVA) at a 10:1 ratio of polymer to iron and a 45:1 ratio of PVA to aminoPVA copolymer (by mass), resulting in USPIO NPs of an hydrodynamic diameter of 25-30 $\mathrm{nm}$ and a zeta potential of $+25 \mathrm{mV}$. The iron content of the preparations of aminoPVA-coated USPIO NPs and oleic acid-coated USPIO NPs was determined by quantitative Prussian blue reaction according to a previously described protocol. ${ }^{15,16}$ The size of aminoPVA USPIO NPs size was determined in DMEM plus FCS cell culture medium by turbidity and photon correlation spectroscopy (PCS) ${ }^{17}$ showing that their size remained stable at their initial $40 \mathrm{~nm}$ for up to 9 days in this medium. No agglomeration was observed. AminoPVA-coated and oleic acid-coated USPIO NPs in suspension in water were diluted immediately before use in cell culture medium and then serially diluted in cell culture medium.

Uncoated USPIO NPs immediately agglomerate in culture medium containing FCS, resulting in agglomerates of large dispersion size with an average size of $910 \mathrm{~nm}$, as determined by DLS and electron microscopy. ${ }^{16}$ Preliminary experiments in the absence of cells (empty Transwells) using Transwell devices of different membrane pore size and composition have shown that these agglomerates are trapped on and in the membrane, and cannot be transported even in the absence of cells or in the presence of a strong dynamic magnetic field. Thus these USPIO NPs were not evaluated in the present report.

\section{Cell models and culture conditions}

Human HT-29 and Caco-2 colon carcinoma cells from the American Type Culture Collection (ATCC, Manassas, VA) were grown in Dulbecco's modified Eagle's medium (DMEM) containing $4.5 \mathrm{~g} / 1$ glucose, 10\% FCS, and antibiotics as monolayer cultures (all cell culture reagents were 
purchased from Gibco, Invitrogen, Basel, Switzerland). For three-dimensional cell cultures (spheroids), HT-29 or Caco- 2 cells were grown in $75 \mathrm{~cm}^{2}$ flasks (Nunclon, Milian, Geneva, Switzerland) until 90\% confluent. Cells were detached in trypsin-EDTA (Gibco) and grown in complete medium in $10 \mathrm{~cm}$ diameter low-adherence bacterial-grade Petri dishes (Sarstedt, Sevelen, Switzerland) to give threedimensional spheroids after 3-4 days of culture. For transport experiments using the CacoReady ${ }^{\mathrm{TM}}$ model (Readycell, Barcelona, Spain), the HT-29 and Caco-2 cells used by the company Advancell (Barcelona, Spain) to develop the barrier model were initially obtained from Dr FX Real (Institut Municipal d'Investigació Mèdica, Barcelona). Caco-2 cells $\left(82.5 \times 10^{4}\right.$ cells $/ \mathrm{cm}^{2}$ in $0.3 \mathrm{~mL}$ of complete medium in the apical upper compartment and $0.9 \mathrm{~mL}$ of complete medium in the basal chamber of Transwells ${ }^{\circledR}$ inserts) were cultured on 24-well polycarbonate filters (Costar, polycarbonate membrane, pore diameter $=3 \mu \mathrm{m}, 24$ inserts per plate) for 14 days in DMEM $1 \mathrm{~g} / 1$ glucose supplemented with $10 \%$ FCS, $2 \mathrm{mM}$ glutamine, $1 \mathrm{U} / \mathrm{mL}$ penicillin, and $1 \mathrm{U} / \mathrm{mL}$ streptomycin, then for 4 days of culture in semi-solid shipping medium, and again for 3 days in regular culture medium (for a total of 21 days of culture). For the CacoGoblet ${ }^{\mathrm{TM}}$ model (Readycell, Barcelona, Spain), a mix of equal volumes of Caco-2 and HT-29-M6 goblet cells (metothrexate-adapted HT-29-M6 clone) were cultured under the same conditions.

\section{Cell uptake of USPIO NPs}

Cells were grown in 48-well plates (Costar) until 75\% confluent, then exposed to USPIO NPs at the concentration and for the time indicated. The plates were washed three times with saline $(0.9 \% \mathrm{NaCl}(\mathrm{w} / \mathrm{v}))$ and cell-associated iron quantified as previously described. ${ }^{14-16}$ Briefly, to quantify cellular iron content cell layers were dissolved at room temperature for 1 hour in $6 \mathrm{~N} \mathrm{HCl}(125 \mu \mathrm{L} /$ well of a 48 -well plate), then $125 \mu \mathrm{L}$ of a $5 \%$ solution of $\mathrm{K}_{4}\left[\mathrm{Fe}(\mathrm{CN})_{6}\right] \cdot 3 \mathrm{H}_{2} \mathrm{O}$ (Merck, VWR international, Nyon, Switzerland) in $\mathrm{H}_{2} \mathrm{O}$ was added for 10 minutes, and the absorbance was read at $690 \mathrm{~nm}$ in a multiwell plate reader (iEMS Labsystems, BioConcepts, Allschwil, Switzerland). A standard curve of iron chloride in $6 \mathrm{~N} \mathrm{HCl}$ treated under the same conditions was used to quantify the amount of cell-bound iron. All experiments were performed in triplicate wells, and repeated at least twice.

On the day of the experiment, the spheroids were centrifuged for five minutes at $1000 \mathrm{rpm}$ at room temperature and gently mixed with fresh complete medium containing aminoPVA USPIO NPs (final iron concentration $=21 \mu \mathrm{g} / \mathrm{mL}$ ) or oleic acid-coated USPIO NPs (final iron concentration $=45 \mu \mathrm{g} / \mathrm{mL}$ ), and incubated with the USPIO NPs in a $10 \mathrm{~cm}$ diameter dish for either 2 hours or 24 hours, centrifuged for 5 minutes at $1000 \mathrm{rpm}$, washed twice with $5 \mathrm{~mL}$ phosphate-buffered saline, and then centrifuged for 5 minutes at $1000 \mathrm{rpm}$. The spheroid pellet was fixed in 4\% buffered formaldehyde (Merck, Darmstadt, Germany) for 30 minutes at room temperature, centrifuged for 10 minutes at $1000 \mathrm{rpm}$, and then fixed in the cell block preparation system (Shandon Cytoblock ${ }^{\circledR}$ Kit, Thermo Scientific, Pittsburgh, PA), centrifuged for 5 minutes at $1500 \mathrm{rpm}$ (Cytospin 3, Shandon), dehydrated and embedded in paraffin, and then histological slides $(5 \mu \mathrm{m})$ were prepared.

\section{Histochemical determination of iron}

After exposure to USPIO NPs the cell layers or the spheroids were washed with saline, fixed in $4 \%$ buffered paraformaldehyde at $4^{\circ} \mathrm{C}$ for 30 minutes, and incubated for 20 minutes at room temperature with a 1:1 solution of $1 \mathrm{M} \mathrm{HCl}$ and $10 \% \mathrm{~K}_{4}\left[\mathrm{Fe}(\mathrm{CN})_{6}\right] \cdot 3 \mathrm{H}_{2} \mathrm{O}$ in $\mathrm{H}_{2} \mathrm{O}$, washed with distilled water, counterstained with Nuclear Fast Red, dehydrated in graded ethanol to xylol, and mounted. Slides were photographed under a Nikon digital camera (DXM 1200; Nikon Corporation, Tokyo, Japan).

\section{Evaluation of cell viability}

Cells were grown in 48-well cell culture plates (Costar, Corning, NY) until $75 \%$ confluent, exposed to USPIO NPs at the concentration and for the time indicated, then washed in saline. Cell viability was evaluated using the 3,4,5-dimethylthiazol-yl-2,5-diphenyl tetrazolium bromide (MTT) assay (Sigma-Aldrich, Buchs, Switzerland) added to the cells in fresh complete culture medium at a $250 \mu \mathrm{g} / \mathrm{mL}$ final concentration. After 2 hours the supernatant was removed, and the precipitated formazan was dissolved in $0.1 \mathrm{~N} \mathrm{HCl}$ in isopropanol and quantified at $540 \mathrm{~nm}$ in a multiwell plate reader (iEMS Labsystems).

\section{Transmission electron microscopy and elemental analysis}

At the end of exposure to the USPIO NPs, the cells were washed twice with phosphate-buffered saline, fixed in $2 \%$ glutaraldehyde buffered in $0.1 \mathrm{M}$ cacodylate for 1 hour at $4^{\circ} \mathrm{C}$, then washed in $0.2 \mathrm{M}$ cacodylate at $4^{\circ} \mathrm{C}$ (three times for 10 minutes), gently scraped in $0.2 \mathrm{M}$ cacodylate, and harvested by centrifugation ( 5 minutes at $1000 \mathrm{rpm}$ ). The pellets were coated with agarose (type III low gelling temperature, Sigma) and cut in small pieces. The samples were postfixed in 1\%-2\% buffered osmium tetroxide for 1 hour at room temperature, 
washed in $0.2 \mathrm{M}$ cacodylate (twice for 5 minutes), dehydrated in graded ethanol (twice for 10 minutes in 70\% ethanol, twice for 10 minutes in $90 \%$ ethanol, and three times for 20 minutes in $100 \%$ ethanol), dehydrated again in propylene oxide (twice for 30 minutes), embedded in equal volumes of propylene oxide and Epon (50\% Epon 812 substitute, 26\% dodecenylsuccinic anhydride, 23\% methyl nadic anhydride, 1\% 2,4,6-tris(dimethylaminomethyl)phenol) (all figures w/w, all materials from Fluka, Buchs, Switzerland) for 1 hour, and then for 16 hours in Epon.

Specimens were embedded in Epon blocks that were cured for 48 hours at $60^{\circ} \mathrm{C}$, and then trimmed and cut into $100 \mathrm{~nm}$ sections and mounted on slides for post-visualization by methylene blue/azur blue staining. Thin sections (50 nm) were cut from the cured blocks using an ultramicrotome (Ultracut E, Reichert-Jung Optische Werke AG, Wien, Austria) and mounted on 3-mm 200-mesh copper grids. Grids were heavy-metal stained using a standard two-step uranyl acetate/lead citrate technique (Leica EM Stain) and then examined and photographed at $80 \mathrm{kV}$ with a Philips CM12 transmission electron microscope (Amsterdam, The Netherlands) combined with a MegaView III, Soft Imaging System (Gloor Instruments, Uster, Switzerland). Elemental analysis of iron was performed by an electron detection X-ray (EDAX, EDX-4), coupled to the CM12 transmission electron microscope.

\section{USPIO NPs transport}

Evaluation of the transport of USPIO NPs by CacoReady and CacoGoblet was performed after 21 days of culture when the cells were completely differentiated as assessed by transepithelial electrical resistance measurements. Both the apical and basal chambers were washed twice with $\mathrm{Ca}^{2+} /$ $\mathrm{Mg}^{2+}$ Hank's balanced salt solution (HBSS) buffer (Gibco) supplemented with $5 \mathrm{mM}$ glucose. USPIO NPs $(100 \mu \mathrm{g} / \mathrm{mL}$ or $70 \mu \mathrm{g} / \mathrm{mL}$ iron) diluted in HBSS buffer were applied to the apical chambers while basal chambers were filled with HBSS buffer without USPIO NPs. After 0, 2, 6, and 24 hours of incubation, $50 \mu \mathrm{L}$ of medium was removed from the basal chambers and analyzed for iron content with the Prussian blue reaction (see above). The volume in the basal chamber was maintained by adding $50 \mu \mathrm{L}$ of fresh medium after each sampling point. Parallel experiments were performed in the absence of cells (empty membranes). The effect of USPIO NPs on CacoReady and CacoGoblet barriers was assessed by Lucifer yellow permeability measurement after the end of the assay (24 hours of exposure to USPIO NPs). Lucifer Yellow-HD (Sigma-Aldrich) solution at a final concentration of $200 \mu \mathrm{M}$ in HBSS buffer was added to the apical chamber and incubated for 1 hour at $37^{\circ} \mathrm{C}$, and $100 \mu \mathrm{L}$ of the medium in the basal compartment was analyzed in a multiwell plate fluorescence reader at $485 \mathrm{~nm} / 530 \mathrm{~nm}$ excitation/emission wavelength, respectively.

\section{Statistical analysis}

Each experiment was repeated in triplicate wells at least twice. Means and standard deviations (SDs) were calculated, and statistical significance was assessed using Student's t-test.

\section{Results \\ Uptake of USPIO NPs by monolayers of human colon cells}

Previous research has shown that positive charges at the surface of aminoPVA-USPIO NPs are necessary for their uptake by cells, including human cells, since aminoPVA-coated (but not carboxylate-PVA-coated or PVA-coated) USPIO NPs were internalized by the cells. ${ }^{14,16}$ Therefore, the association of these previously described cationic aminoPVA USPIO NPs (Figure 1) with HT-29 and Caco-2 human colon cells was compared to their association with anionic oleic acid-coated USPIO NPs (Figure 2), as determined through the evaluation of cell-associated iron by histological or quantitative Prussian blue reaction. In both cell lines, the association of aminoPVA USPIO NPs dose-dependently increased with time (Figure 1), while anionic USPIO NPs were only poorly associated with colon cells and this association did not increase with longer exposure time (Figure 2). For these USPIO NPs, the iron content in the cell layers was below the detection limit of the Prussian blue method. Therefore, cationic USPIO NPs associated with human colon cells to a larger extent than anionic USPIO NPs. Similar results were obtained in both cell lines (data of the histological Prussian blue reaction are not shown for HT-29 cells).

An MTT test was used to evaluate cell metabolic activity to determine whether uptake resulted in a cytotoxic effect for the cells. Neither aminoPVA nor oleic acid-coated USPIO NPs inhibited cell metabolic activity except for the highest concentrations of oleic acid-coated USPIO NPs tested (Figure 3A and B) in both cell lines after 24 hours of exposure.

It was demonstrated through the use of transmission electron microscopy that the iron oxide core of these aminoPVA USPIOs was localized in granular structures inside HT-29 cells, with microscopy performed after either 6 hours (Figure 4A) or 24 hours (Figure 4B). The presence 

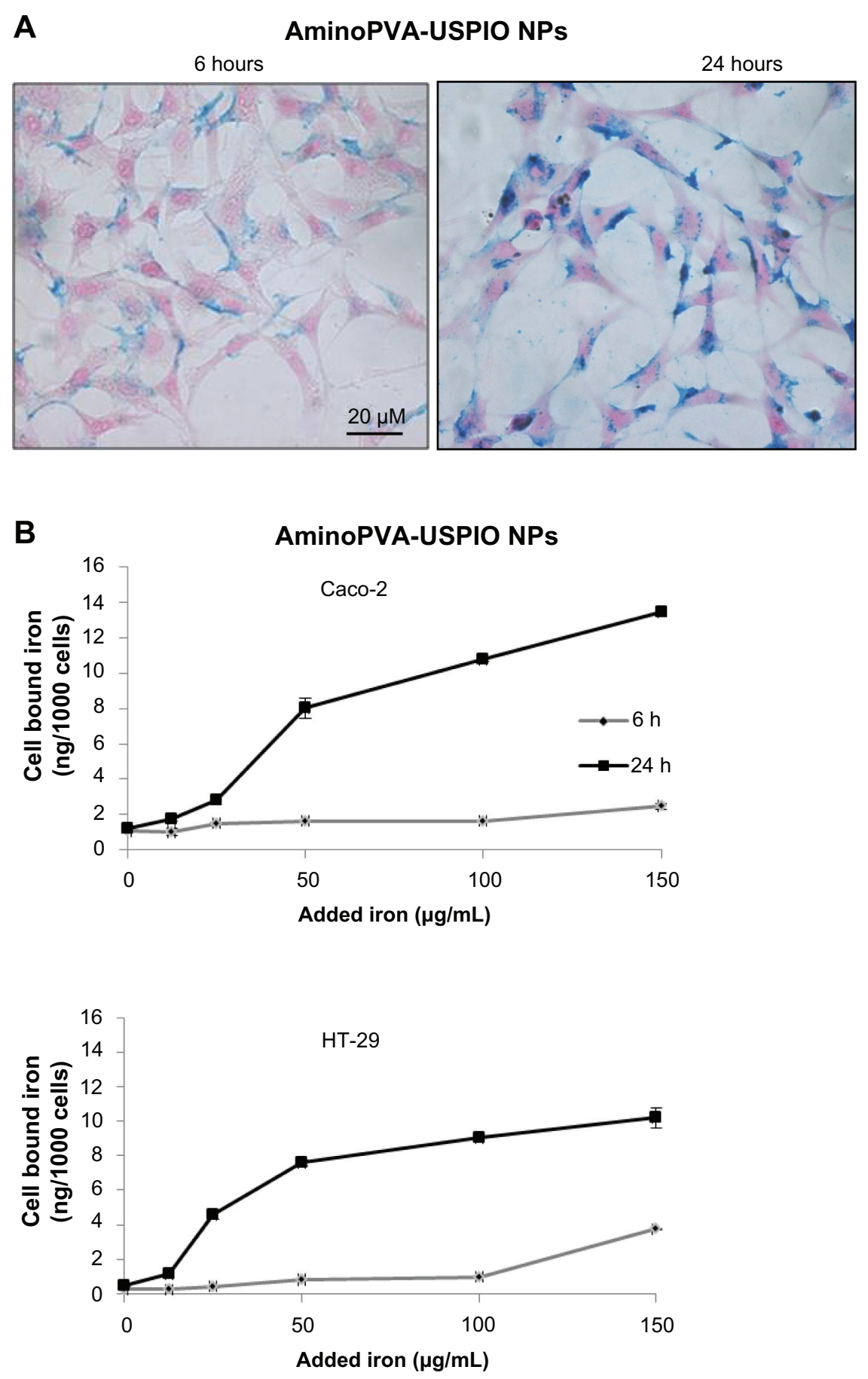

Figure I Time-dependent uptake of aminoPVA-coated USPIO NPs by Caco-2 and HT-29 cells. (A) Caco-2 cells were grown to $75 \%$ confluence, then were exposed for 6 or 24 hours in complete culture medium to $20 \mu \mathrm{g} / \mathrm{mL}$ aminoPVA USPIO NPs. Then cells were stained with Prussian blue and Nuclear Red histological stains (iron blue, nucleus red, cytoplasm pink). (B) Caco-2 and HT-29 cells were grown to $90 \%$ confluence, then they were exposed for 6 hours (grey line) or 24 hours (black line) in complete culture medium to increasing concentrations of aminoPVA USPIO NPs and the cell-associated iron content of the cell layer was quantified using the Prussian blue reaction. Abbreviations: aminoPVA, polyvinylamine; USPIO NPs, ultrasmall superparamagnetic iron oxide nanoparticles.

of iron was confirmed after 24 hours by elemental analysis (Figure 4D), and it was found that more iron was associated with HT-29 cells and that this was located closer to the nucleus after 24 hours. For the purpose of comparison, cell-associated iron was quantified by quantitative Prussian blue reaction (Figure 4C).

\section{Uptake of USPIO NPs by spheroids of human colon cells}

HT-29 cells form well-structured spheroids. After 2 or 24 hours' exposure, it was determined whether aminoPVA USPIO NPs have the potential to invade three-dimensional spheroids of HT-29. The results showed that the iron oxide core of very 


\section{A}

\section{Oleic acid-USPIO NPs}
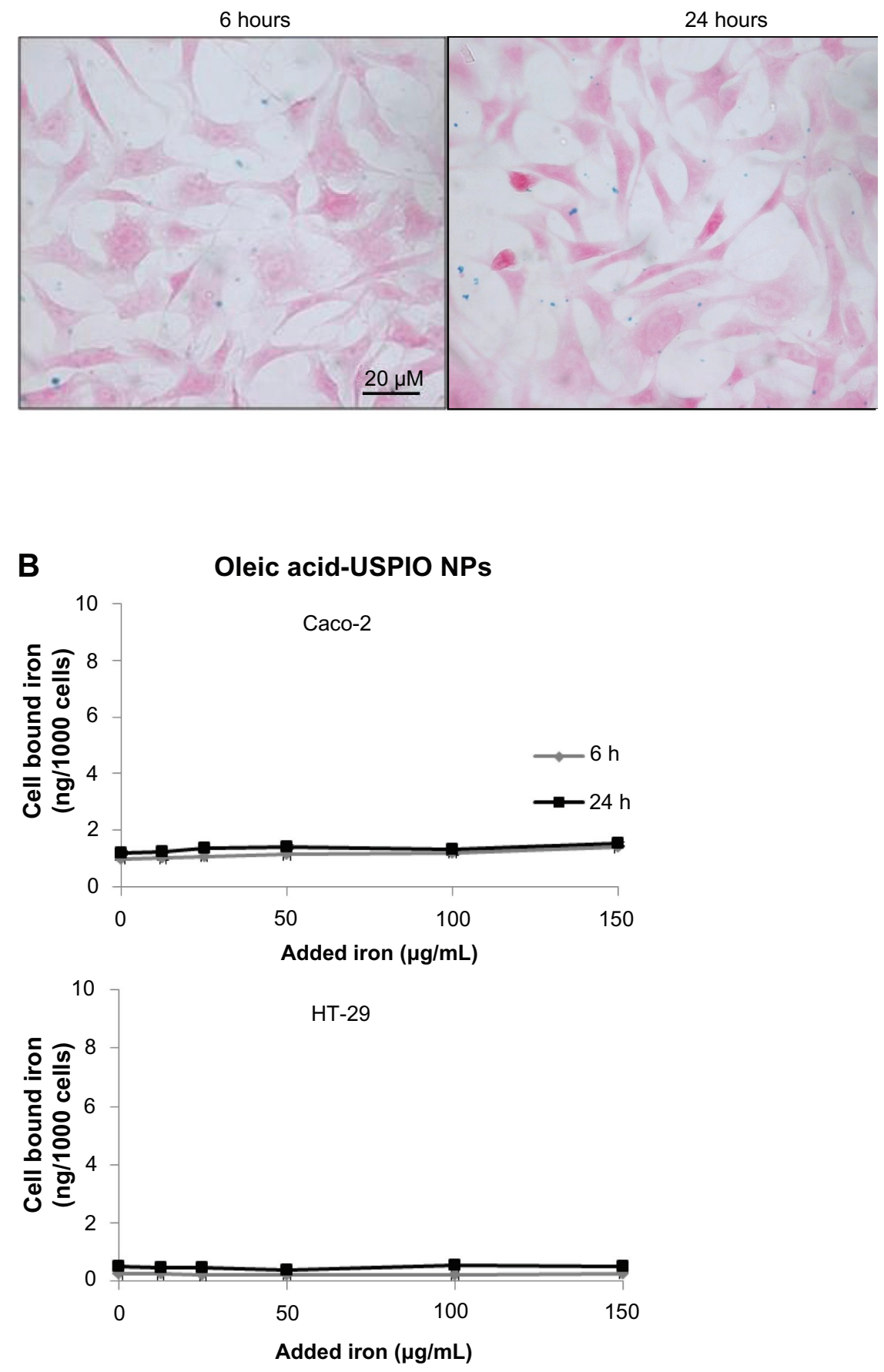

Figure 2 Time-dependent uptake of oleic acid-coated ultrasmall superparamagnetic iron oxide nanoparticles (USPIO NPs) by Caco-2 and HT-29 cells. (A) Caco-2 cells were grown to $75 \%$ confluence, then were exposed for 6 or 24 hours in complete culture medium to $20 \mu \mathrm{g} / \mathrm{mL}$ oleic acid-coated USPIO NPs. Then cells were stained with Prussian blue and Nuclear Red histological stains (iron blue, nucleus red, cytoplasm pink). (B) Caco-2 and HT-29 cells were grown to $90 \%$ confluence, then they were exposed for 6 hours (grey line) or 24 hours (black line) in complete culture medium to increasing concentrations of oleic acid-coated USPIO NPs and the cell-associated iron content of the cell layer was quantified using the Prussian blue reaction.

few aminoPVA USPIO NPs could be detected by the Prussian blue reaction at the surface of the spheroids after 2 hours' exposure, whereas after 24 hours' exposure a large number of aminoPVA USPIO NPs could be demonstrated at the surface as well as in deeper layers of the spheroids (Figure 5A). This demonstrated their potential to invade three-dimensional colon tissue structures.

The invasion of spheroids of either HT-29 or Caco-2 cells was compared after 24 hours by aminoPVA USPIO NPs and oleic acid-coated USPIO NPs (Figure 5B). Caco-2 cells 

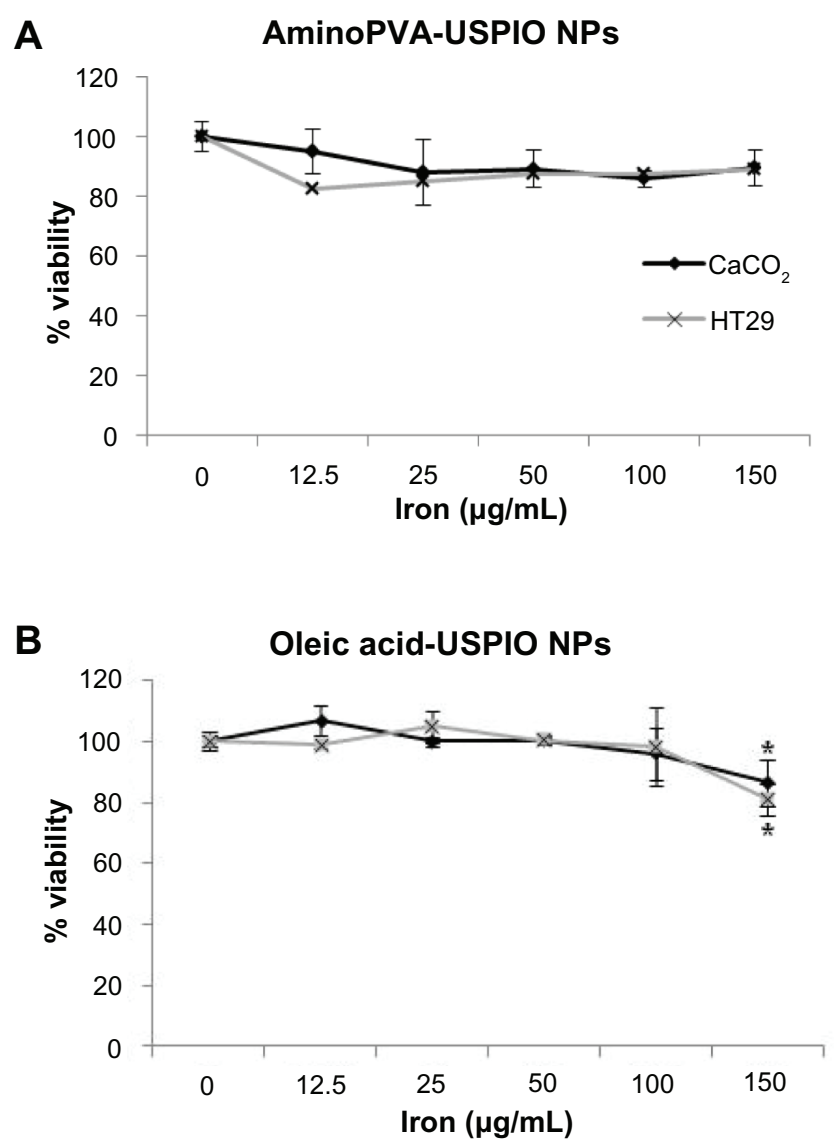

Figure 3 Cytotoxicity of aminoPVA- and oleic acid-coated USPIO NPs for colon carcinoma cells. Caco-2 cells (black line) and HT-29 cells (grey line) were exposed for 24 hours to aminoPVA-coated (A) and oleic acid-coated (B) USPIO NPs, then an MTT test was performed to ascertain cytotoxicity.

Notes: Means \pm standard deviation were calculated, and statistical significance was assessed using Student's $t$-test; $* P<0.05$, exposed cells compared to control cells Abbreviations: aminoPVA, polyvinylamine; USPIO NPs, ultrasmall superparamagnetic iron oxide nanoparticles.

form smaller and less cohesive spheroids than HT-29 cells. Whereas the presence of the iron oxide core of aminoPVA USPIO NPs was observed inside spheroids of both cells, the presence of the iron oxide core of oleic acid-coated USPIO NPs was observed only in spheroids of Caco-2 cells.

\section{Transport of USPIO NPs by colon cells}

The ability of two different cellular experimental models of the gastrointestinal barrier (the tight CacoReady model and the more permeable mucus-secreting CacoGoblet model) to transport cationic and anionic USPIO NPs was evaluated. Cells were exposed to the USPIO NPs for 24 hours after which LY permeability was assessed, and this demonstrated that the presence of USPIO NPs did not affect the integrity of the CacoReady and CacoGoblet barriers (results not shown). The transport experiments performed for a 24-hour time-course showed that whereas the USPIO NPs were transported across empty Transwell membranes, neither gastrointestinal barrier models efficiently transported the USPIO NPs (Figure 6). The noncumulated amount of USPIO NPs in the basal compartment of the device showed that oleic acid-coated USPIO NPs transfer less across the membrane than the aminoPVA USPIO NPs, with most of the transfer of oleic acid-coated USPIO NPs being achieved within 2 hours.

\section{Discussion}

The success of a nanoparticle-based therapy depends largely on the behavior of the NPs in the tissue microenvironment and their interactions with cells forming the tissue. The present study focused on the mechanisms of uptake and transport of cationic and anionic ultrasmall superparamagnetic iron oxide NPs (USPIO NPs) by human colon cells. USPIO NPs composed of a $9 \mathrm{~nm}$ monocrystalline core coated with polyvinylamine/polyvinylalcohol (aminoPVA USPIO NPs) for biological compatibility and stability had previously been developed. ${ }^{13,14}$ These cationic aminoPVA USPIO NPs with a defined number of positive amino groups at their surface have been shown to have the potential to be internalized by cells with their polymers ${ }^{15}$ even when the polymers were chemically functionalized with anti-cancer drugs, ${ }^{18,19}$ and that internalization was dependent on the positive charges at their surface. In the current study, the behavior toward human colon cancer cells of these cationic aminoPVA USPIO NPs was compared with anionic oleic acid-coated USPIO NPs with comparable core and hydrodynamic sizes. Whereas human colon cells efficiently internalized the cationic USPIO NPs in a time- and concentration-dependent manner, the uptake of the anionic USPIO NPs was very low (to the extent that it was hardly detectable by the Prussian blue method) and did not increase with longer exposure times. Electron microscopy images of HT-29 cells suggested that aminoPVA USPIO NPs were localized intracellularly in the endosomal/lysosomal compartment and that they retained their initial nanometric dimension for 24 hours. A number of recent in vitro and in vivo studies highlight the importance of NP surface charge for cellular uptake and biodistribution, ${ }^{20-22}$ indicating that for most particles a positive charge at their surface enhances cellular internalization, ${ }^{22-24}$ which is very likely linked to the adsorption of different biological molecules at the surface of NPs (the corona) dependent on surface charge and chemical characteristics. ${ }^{12}$

Another important issue when considering NPs for therapeutic purposes is their potential to penetrate tissues. Three-dimensional cell culture models have been developed in order to mimic tissue structure better than is possible with two-dimensional monolayer cell cultures. Three-dimensional 


\section{AminoPVA-USPIO NPs - HT-29 cells}

A

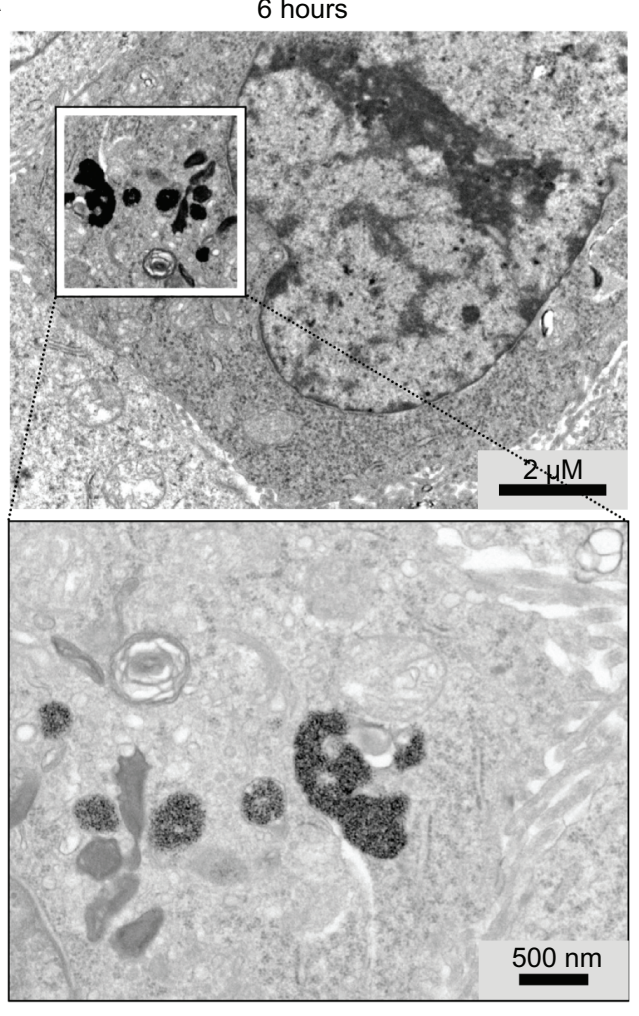

C

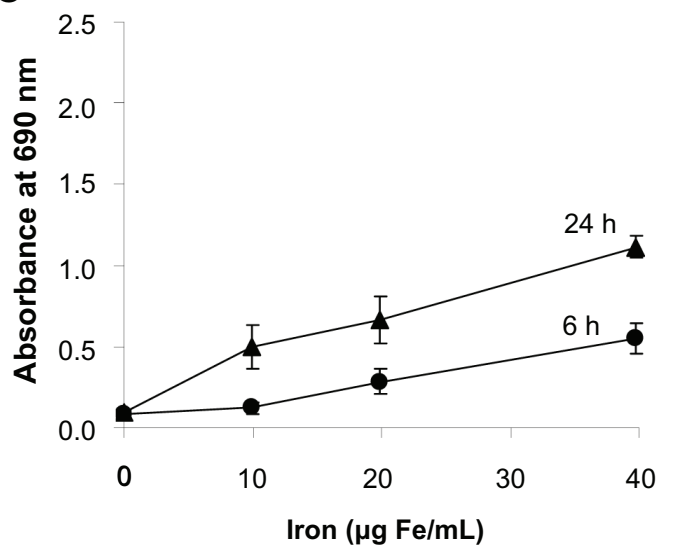

B
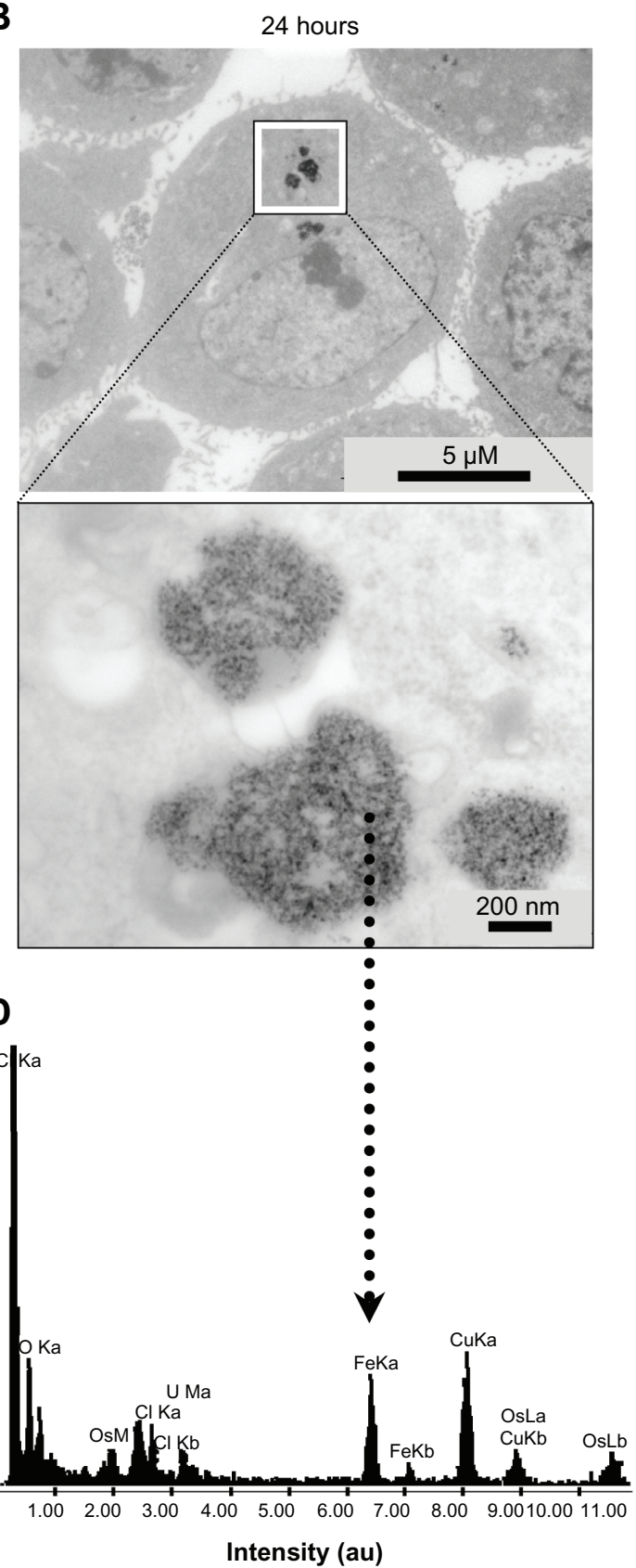

Figure 4 Cellular localization of aminoPVA-coated USPIO NPs in HT-29 cells. (A) After 6 hours of exposure, transmission electron microscopy images demonstrated that the iron oxide core of aminoPVA USPIO NPs $(21 \mu \mathrm{g} \mathrm{Fe} / \mathrm{mL})$ was localized in intracellular organelles of HT-29 cells. (B) After 24 hours of exposure the iron oxide core of aminoPVA USPIO NPs (2I $\mu \mathrm{g} \mathrm{Fe} / \mathrm{mL})$ was localized closer to the nucleus. (C) For the purpose of comparison, cell-associated iron was quantified by quantitative Prussian blue reaction. (D) Elemental analysis (dotted arrow) performed confirmed the presence of iron in the area defined by the black square of the transmission electron microscopy images (lower panel, right).

Abbreviations: aminoPVA, polyvinylamine; USPIO NPs, ultrasmall superparamagnetic iron oxide nanoparticles.

cell models, such as normal cell aggregates or tumor spheroids, allow researchers to study drug penetration and cell-cell interactions. ${ }^{25-29}$ It has previously been shown that aminoPVA USPIO NPs localize exclusively in the first layer of nontumoral three-dimensional aggregates of differentiated rat brain cells,${ }^{15}$ whereas NPs were able to penetrate into deeper layers of spheroids of human melanoma cells, likely through the use of vasculogenic mimicry structures. ${ }^{30}$

In the present study, a three-dimensional spheroid model system was obtained by growing HT-29 or Caco- 2 cells under static conditions which resulted in rounded, compacted, and regularly shaped spheroids, for all spheroids, however, 
A
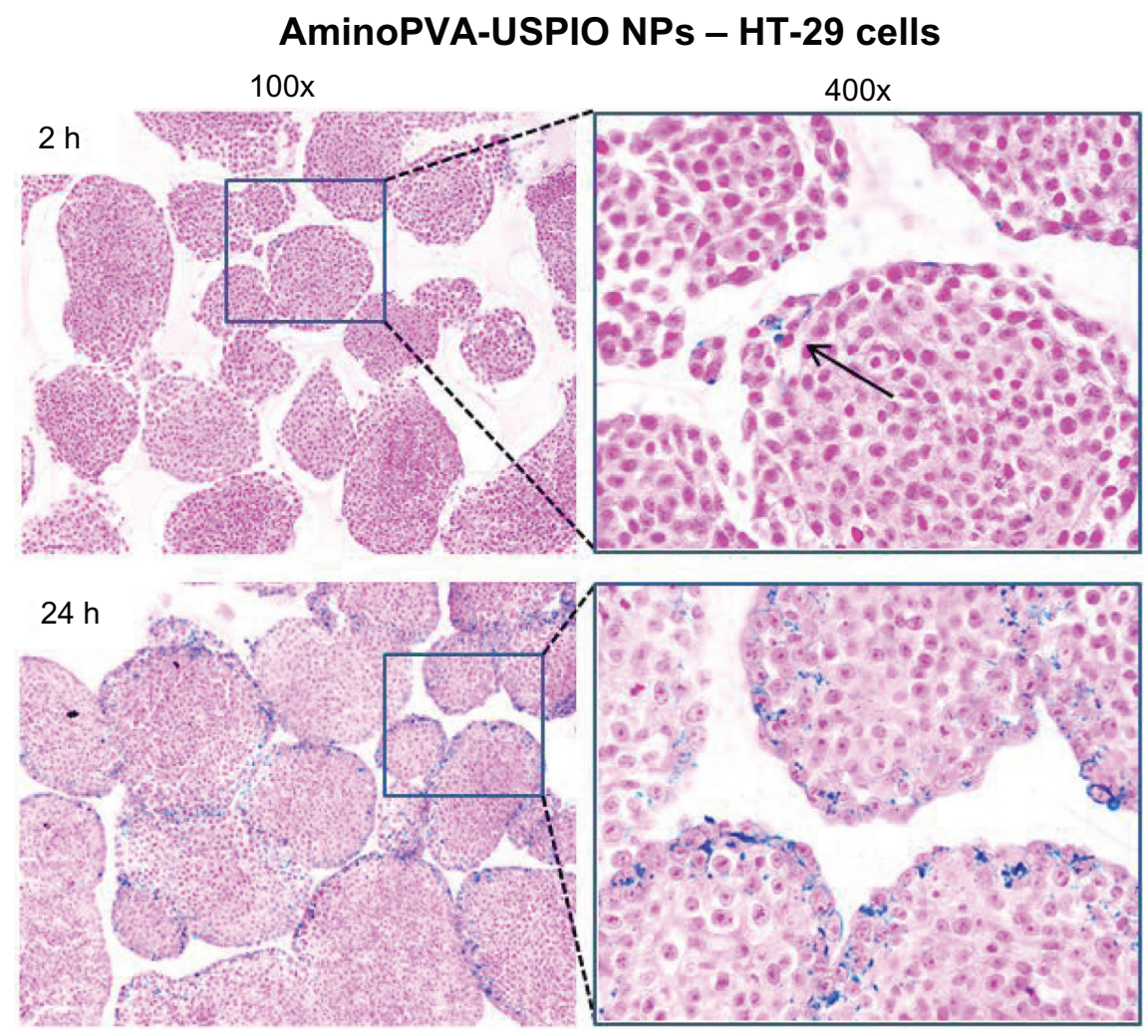

B

AminoPVA-USPIO NPs
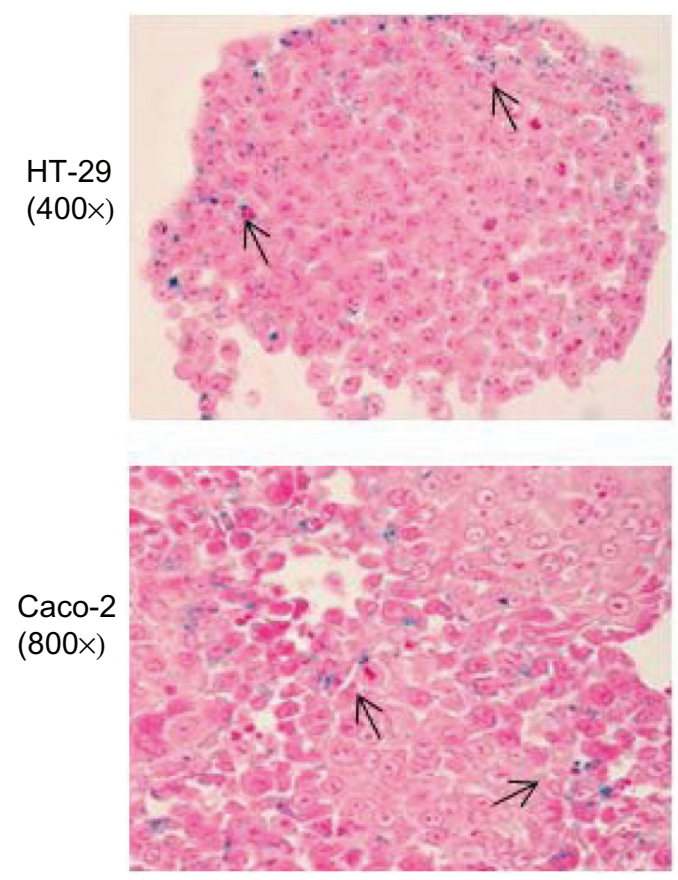

Oleic acid-USPIO NPs
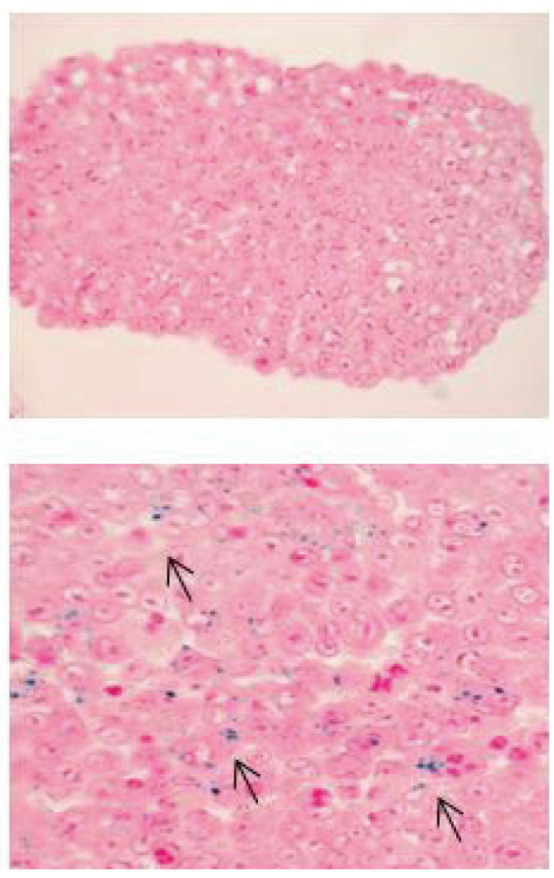

Figure 5 Polyvinylamine (aminoPVA)- and oleic acid-coated ultrasmall superparamagnetic iron oxide nanoparticles (USPIO NPs) selectively invade three-dimensional spheroids of human HT-29 and Caco-2 colon carcinoma cells. (A) Human HT-29 cells in three-dimensional spheroid cultures form well-structured spheroids after 3 days. The spheroids were exposed to aminoPVA USPIO NPs ( $21 \mu \mathrm{g} \mathrm{Fe} / \mathrm{mL}$ ) for 2 or 24 hours, recovered, fixed, then cell-associated iron was determined on histological slides by the histological Prussian blue reaction (iron, blue staining; cell cytoplasm, pink; cell nuclei, red). After 2 hours aminoPVA USPIO NPs were found in very few cells (arrow) of the first layer of the spheroids, but after 24 hours' exposure of the spheroids they were more widely found in many cells, including in cells localized more deeply in the spheroids. (left, I00×; right, $400 \times$ magnification). (B) Human HT-29 and Caco-2 cells in three-dimensional spheroid cultures were exposed to aminoPVA-coated USPIO NPs ( $21 \mu \mathrm{g}$ iron/mL, left panels) and oleic acid-coated USPIO NPs (45 $\mu \mathrm{g}$ iron/mL, right panels) for 24 hours, recovered, fixed, then cell-associated iron (arrows) was determined on histological slides by the histological Prussian blue reaction (iron, blue staining; cell cytoplasm, pink; cell nuclei, red). 
A

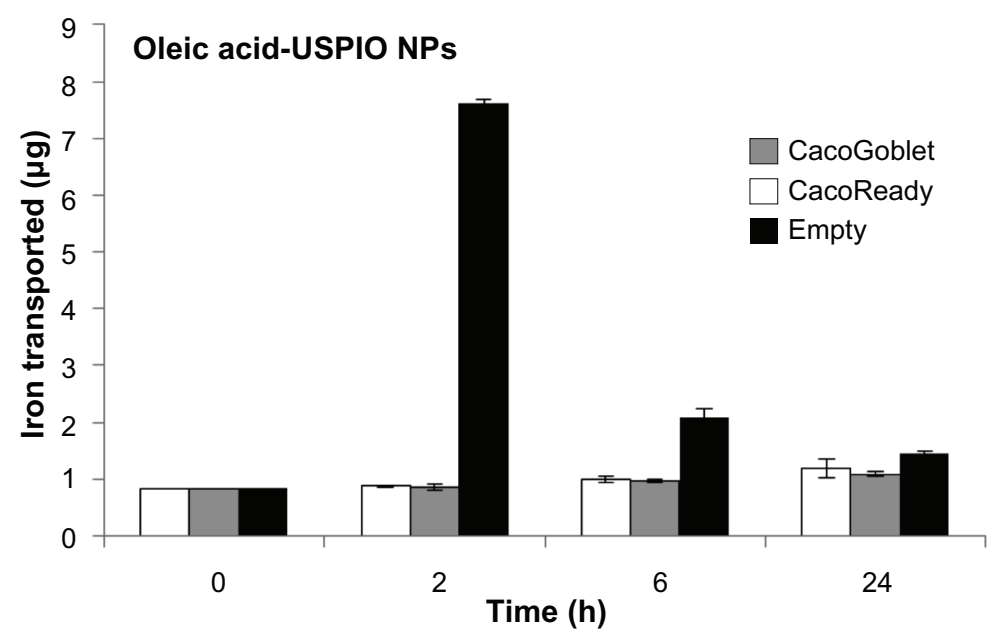

B

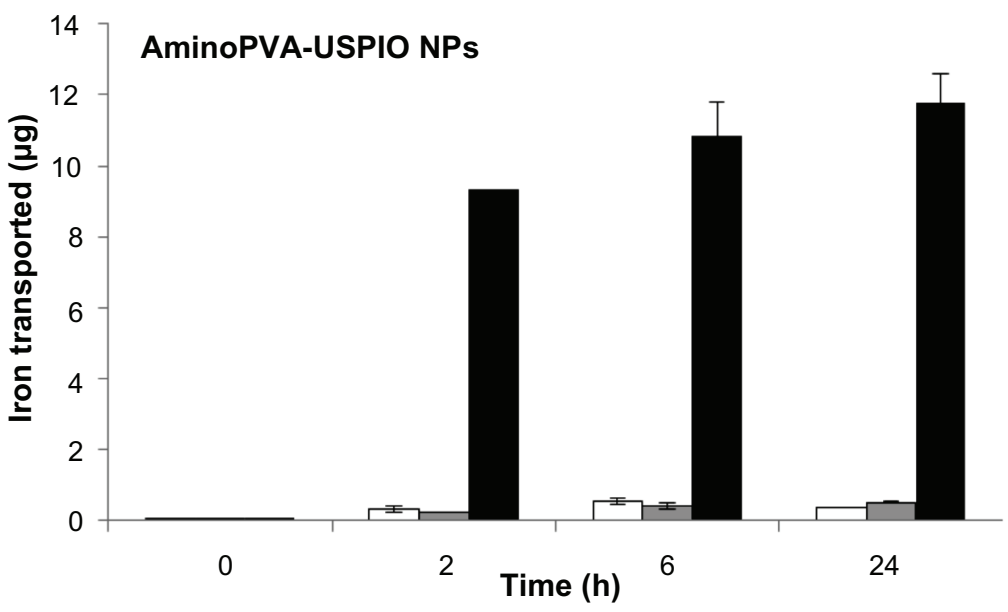

Figure 6 Transport of ultrasmall superparamagnetic iron oxide nanoparticles (USPIO NPs) by human colon cells. Transport of polyvinylamine (aminoPVA) USPIO NPs (A) and oleic acid-coated USPIO NPs (B) across CacoReady ${ }^{\mathrm{TM}}$ (white bars) and CacoGoblet ${ }^{\mathrm{TM}}$ (grey bars) colon barriers and across empty membrane (black bars). The initial concentration of iron in apical chamber was $100 \mu \mathrm{g} / \mathrm{mL}(\mathbf{A})$ or $70 \mu \mathrm{g} / \mathrm{mL}(\mathbf{B})$. Iron concentration in the basal chamber was quantified with the Prussian blue reaction. Note: The results represent noncumulated amounts of iron in the basal compartments at each time-point, without addition of USPIO NPs in the apical compartment.

spheroids from Caco-2 cells were less well structured and less cohesive than spheroids from HT-29 cells. Penetration of HT-29 cell spheroids was limited to the first cell layers after 2 hours of exposure to aminoPVA USPIO NPs, but after 24 hours of exposure the aminoPVA USPIO NPs were localized in the lower layers of the spheroids. The aminoPVA USPIO NPs were also able to invade deep layers of Caco-2 cell spheroids after 24 hours of exposure. However, oleic acid-coated USPIO NPs could only be found in spheroids of Caco-2 cells, which were generally smaller and less cohesive than HT-29 spheroids. Thus, the uptake by the spheroids of oleic acid-coated USPIO NPs was dependent on the cancer cells considered. Therefore, for interactions with biological structures both the surface properties of NPs and the characteristics of the tissue are important.

However, neither aminoPVA-coated nor oleic acidcoated USPIO NPs were transported across gastrointestinal barriers such as the CacoReady (Caco-2 cells only) or the CacoGoblet (Caco-2 cells and HT-29-M6 cells in co-culture) colon barrier models. CacoReady represents a tight barrier model while CacoGoblet represents a more permeable mucus-secreting barrier model that mimics normal physiological conditions. The differentiated Caco-2 cells alone or in co-culture are well characterized cellular models of 
normal intestinal epithelium..$^{31,32}$ Monolayers of these cells have been widely used to study the unidirectional transport of diverse drugs and particles from the apical to the basolateral side, and these results correlate with in vivo experiments. ${ }^{33,34}$ The major mechanism for drug uptake across the intestinal mucosa is passive diffusion where the driving force is a concentration gradient. The ionization state of the agents is important for transport. The transport of the drug valdecoxib entrapped in microspheres was determined using this model, but only the drug was analyzed in the basolateral side of the Transwell, and the authors postulated drug release prior to transport. No attempt was made to evaluate whether the microspheres were transported by the cells. ${ }^{35}$ The current study shows that neither cationic aminoPVA USPIO NPs nor anionic oleic acid-coated USPIO NPs were transported across these colon barriers during a 24-hour evaluation. Thus, the ability of NPs to penetrate three-dimensional tissue does not predict their potential to cross cellular barriers such as the intestinal barrier. In the few available studies dealing with transport issues only a small number of the tested NPs (such as lipid nanocapsules or chitosan NPs) were successfully transported across the intestinal barrier model in vitro. ${ }^{36-38}$ The mobility of polystyrene NPs in the gastrointestinal mucus was shown to be strongly dependent on their surface charge and chemistry. ${ }^{39}$ The interactions of uncoated, aminoPVA-coated, and oleic acid-coated USPIO NPs have been evaluated with human brain endothelial cells as a blood-brain barrier (BBB) model, with this work showing that while the cells could internalize the NPs, none were transported across the BBB model, even in the presence of a strong magnetic field. ${ }^{16}$ Thus, the BBB and the gastrointestinal barrier models provided comparable information regarding USPIO NPs transport.

\section{Conclusion}

USPIO NPs are useful MRI agents in vivo, and when functionalized with therapeutic or diagnostic agents - the so-called theranostic agents - the distribution of theranosticsUSPIO NPs in a living human will provide information on the biodistribution of the theranostics and their ability to reach their targets. Thus, the present project aimed to evaluate the surface characteristics of USPIO NPs that allow or prevent their uptake by and transport across human colon cells. This would allow their potential as drug-delivering agents to be evaluated, as well as their potential for invading three-dimensional models of human colon cells, which better mimic the tissue structures of normal and tumoral colon than the two-dimensional models used in the past.
The results presented here show that cationic USPIO NPs are more promising than anionic USPIO NPs for their intracellular delivery into human colon cells, but these NPs will not cross an intact intestinal barrier whatever their charge. Nevertheless, depending on their surface charge and the physiological characteristics of the threedimensional tissue, they have the potential to invade three-dimensional spheroids of human colon carcinoma cells (which act as models of human colon cancer). These observations are important for the development of either therapeutic and theranostic NPs for the extracellular or intracellular delivery of therapeutic agents, or of diagnostic NPs for the imaging of diseases of the human gastrointestinal tract.

\section{Acknowledgments}

This work was supported by grants from the Swiss National Scientific Research Foundation (grant no. 3152 A0-105705), the Swiss League and Research against Cancer (grant no. KLS-01308-02-2003) and the European Community FP7 project "NanoTEST" (grant no 2007-201335).

\section{Disclosure}

The authors declare no conflicts of interest in this work.

\section{References}

1. Heath JR, Davis ME. Nanotechnology and cancer. Annu Rev Med. 2008;59:251-265.

2. Caruthers SD, Wickline SA, Lanza GM. Nanotechnological applications in medicine. Curr Opin Biotechnol. 2007;18(1):26-30.

3. Wang YXJ, Hussain SM, Krestin GP. Superparamagnetic iron oxide contrast agents: physicochemical characteristics and applications in MR imaging. Eur Radiol. 2001;11(11):2319-2331.

4. Wu EX, Tang H, Jensen JH. Applications of ultrasmall superparamagnetic iron oxide contrast agents in the MR study of animal models. NMR Biomed. 2004;17(7):478-483.

5. Corot C, Robert P, Idée JM, Port M. Recent advances in iron oxide nanocrystal technology for medical imaging. Adv Drug Delivery Rev. 2006;58(14):1471-1504.

6. Zhang W, Kalive M, Capco DG, Chen Y. Adsorption of hematite nanoparticles onto Caco-2 cells and the cellular impairments: effect of particle size. Nanotechnology. 2010;21(35):355103.

7. Gambarota G, van Laarhoven HW, Philippens M, et al. Assessment of blood hemodynamics by USPIO-induced R(1) changes in MRI of murine colon carcinoma. Appl Magn Reson. 2010;38(3): 349-360.

8. Kumagai M, Sarma TK, Cabral H, et al. Enhanced in vivo magnetic resonance imaging of tumors by PEGylated iron-oxide-gold core-shell nanoparticles with prolonged blood circulation properties. Macromol Rapid Commun. 2010;31(17):1521-1528.

9. Zou P, Yu Y, Wang YA, et al. Superparamagnetic iron oxide nanotheranostics for targeted cancer cell imaging and $\mathrm{pH}$-dependent intracellular drug release. Mol Pharm. 2010;7(6):1974-1984.

10. Verma A, Stellacci F. Effect of surface properties on nanoparticle-cell interactions. Small. 2010;6(1):12-21.

11. Hillaireau H, Couvreur P. Nanocarriers' entry into the cell: relevance to drug delivery. Cell Mol Life Sci. 2009;66(17):2873-2896. 
12. Lundqvist M, Stigler J, Elia G, Lynch I, Cedervall T, Dawson KA. Nanoparticle size and surface properties determine the protein corona with possible implications for biological impact. Proc Nat Acad Sci U S A. 2008;105(38):14265-14270.

13. Chastellain M, Petri A, Hofmann H. Particle size investigations of a multistep synthesis of PVA coated superparamagnetic nanoparticles. J Colloid Interface Sci. 2004;278(2);353-360.

14. Petri-Fink A, Chastellain M, Juillerat-Jeanneret, L, Ferrari A, Hofmann H. Development of functionalized superparamagnetic iron oxide nanoparticles for interaction with human cancer cells. Biomaterials. 2005;26(15):2685-2694.

15. Cengelli F, Maysinger D, Tschudi-Monnet F, et al. Interaction of functionalized superparamagnetic iron oxide nanoparticles with brain structures. J Pharm Exp Therap. 2006;318(1):108-116.

16. Halamoda Kenzaoui, B, Chapuis Bernasconi C, Hofmann H, Juillerat-Jeanneret L. Evaluation of uptake and transport of ultrasmall superparamagnetic iron oxide nanoparticles by human brain-derived endothelial cells. Nanomedicine. 2012;7(1):39-53.

17. Petri-Fink A, Steitz B, Finka A, Salaklang J, Hofmann H. Effect of cell media on polymer coated superparamagnetic iron oxide nanoparticles (SPIONs): colloidal stability, cytotoxicity, and cellular uptake studies. Eur J Pharm Biopharm. 2008;68(1):129-137.

18. Hanessian S, Grzyb JA, Cengelli F, Juillerat-Jeanneret L. Synthesis of chemically functionalized superparamagnetic nanoparticles as delivery vectors for chemotherapeutic drugs. Bioorg Med Chem. 2008;16(6):2921-2931.

19. Cengelli F, Grzyb JA, Montoro A, Hofmann H, Hanessian S, Juillerat-Jeanneret L. Surface-functionalized ultrasmall superparamagnetic nanoparticles as magnetic delivery vectors for camptothecin. Chem Med Chem. 2009;4(6):988-997.

20. He C, Hu Y, Yin L, Tang C, Yin C. Effects of particle size and surface charge on cellular uptake and biodistribution of polymeric nanoparticles. Biomaterials. 2010;31(13):3657-3666.

21. Xiao K, Li Y, Luo J, et al. The effect of surface charge on in vivo biodistribution of PEG-oligocholic acid based micellar nanoparticles. Biomaterials. 2011;32(13):3435-3446.

22. Yue ZG, Wei W, Lv PP, et al. Surface charge affects cellular uptake and intracellular trafficking of chitosan-based nanoparticles. Biomacromolecules. 2011;12(7):2440-2446.

23. Kelf TA, Sreenivasan VK, Sun J, Kim EJ, Goldys EM, Zvyagin AV. Non-specific cellular uptake of surface-functionalized quantum dots. Nanotechnology. 2010;21(28):285105.

24. Chen L, McCrate JM, Lee JC, Li H. The role of surface charge on the uptake and biocompatibility of hydroxyapatite nanoparticles with osteoblast cells. Nanotechnology. 2011;22(10):105708.

25. Mueller-Klieser W. Three-dimensional cell cultures: from molecular mechanisms to clinical applications. Am J Physiol. 1997;273(4 Pt 1):1109-1123.
26. Juillerat-Jeanneret L, Tschudi-Monnet F, Zürich MG, Lohm S, Duijvestijn AM, Honegger P. Regulation of peptidase activity in a three-dimensional aggregate model of brain tumor vasculature. Cell Tissue Res. 2003;311(1):53-59.

27. Sutherland R, Carlsson J, Durand R, Yuhas J. Spheroids in cancer research. Cancer Res. 1981;41:2980-2984.

28. Kunz-Schughart LA. Multicellular tumor spheroids: intermediates between monolayer culture and in vivo tumor. Cell Biol Int. 1999;23(3): 157-161.

29. Santini MT, Rainaldi G. Three-dimensional spheroid model in tumor biology. Pathobiology. 1999;67(3):148-157.

30. Cengelli F, Voinesco F, Juillerat-Jeanneret L. Interaction of cationic ultrasmall superparamagnetic iron oxide nanoparticles with human melanoma cells. Nanomedicine (Lond). 2010;5(7):1075-1087.

31. Delie F, Rubas W. A human colonic cell line sharing similarities with enterocytes, as a model to examine oral absorption: advantages and limitations of the Caco-2 model. Crit Rev Ther Drug Carrier Syst. 1997;14(3):221-286

32. Sambuy Y, De Angelis I, Ranaldi G, Scarino ML, Stammati A, Zucco F. The Caco-2 cell line as a model of the intestinal barrier: influence of cell and culture related factors on Caco-2 cell functional characteristics. Cell Biol Toxicol. 2005;21(1):1-26.

33. Artursson P. Epithelial transport of drugs in cell culture. I. A model for studying the passive diffusion of drugs over intestinal absorptive (Caco-2) cells. J Pharm Sci. 1990;79(6):476-482.

34. Artursson P, Karlsson J. Correlation between oral drug absorption in humans and apparent drug permeability coefficients in human intestinal epithelial (Caco-2) cells. Biochem Biophys Res Commun. 1991;175(3):880-885.

35. Thakral NK, Ray AR, Bar-Shalom D, Eriksson AH, Majumdar DK. The quest for targeted delivery in colon cancer: mucoadhesive valdecoxib microspheres. Int J Nanomedicine. 2011;6:1057-1068.

36. Behrens I, Pena AI, Alonso MJ, Kissel T. Comparative uptake studies of bioadhesive and non-bioadhesive nanoparticles in human intestinal cell lines and rats: the effect of mucus on particle adsorption and transport. Pharm Res. 2002;19(8):1185-1193.

37. Roger E, Lagarce F, Garcion E, Benoit JP. Lipid nanocarriers improve paclitaxel transport throughout human intestinal epithelial cells by using vesicle-mediated transcytosis. J Control Release. 2009;140(2):174-181.

38. Kadiyala I, Loo Y, Roy K, Rice J, Leong KW. Transport of chitosanDNA nanoparticles in human intestinal M-cell model versus normal intestinal enterocytes. Eur J Pharm Sci. 2010;39(1-3):103-109.

39. Crater JS, Carrier RL. Barrier properties of gastrointestinal mucus to nanoparticle transport. Macromol Biosci. 2010;10(12):1473-1483.
International Journal of Nanomedicine

\section{Publish your work in this journal}

The International Journal of Nanomedicine is an international, peerreviewed journal focusing on the application of nanotechnology in diagnostics, therapeutics, and drug delivery systems throughout the biomedical field. This journal is indexed on PubMed Central, MedLine, CAS, SciSearch ${ }^{\circledR}$, Current Contents ${ }^{\circledR} /$ Clinical Medicine,

\section{Dovepress}

Journal Citation Reports/Science Edition, EMBase, Scopus and the Elsevier Bibliographic databases. The manuscript management system is completely online and includes a very quick and fair peer-review system, which is all easy to use. Visit http://www.dovepress.com/ testimonials.php to read real quotes from published authors. 